\title{
PERFORMANCE ANALYSIS AND OPTIMIZATION OF TCP OVER ADAPTIVE WIRELESS LINKS
}

\author{
P. Di Marco, C. Rinaldi, F. Santucci \\ University of L'Aquila \\ Center of Excellence DEWS \\ 67040 Poggio di Roio, L’Aquila, \\ Italy \\ pdimarco@tin.it, $\{$ rinaldi,santucci\}@ing.univaq.it
}

\section{ABSTRACT}

This paper proposes an analytical framework for performance evaluation of TCP (Transport Control Protocol) over adaptive wireless links. Specifically, we include adaptation of power, modulation format and error recovery strategy, and incorporate some features of wireless fading channels. This framework is then used to pursue joint optimization through maximization of an objective functional, that expresses a trade-off between achievable throughput and energy costs. A set of numerical results is reported, and it is seen that Hybrid ARQ schemes may provide significant benefits in the optimization framework.

\section{INTRODUCTION}

One of the most relevant challenges in recent years has concerned efficient deployment of TCP over wireless links, where propagation impairments such as shadowing, multipath propagation and path loss [1] have detrimental effects on transport performance. Indeed, since TCP was designed to work on wired (non fading) links, it tends to react to packet losses by initiating a congestion control algorithm, which causes the reduction in the load of the intermediate links and hence a suboptimal end-to-end performance. While on one side efforts have been devoted to develop analytical setups for performance analysis (see e.g. [2] for characterization of sequences of errors in correlated fading channels), other efforts have been directed to overcome performance degradation. The most relevant solutions to improve the behaviour of TCP over wireless links can be subdivided into three categories: split connection (i.e. [3]), end-to-end (i.e. [4, 5]), and link layer approach. We are mainly interested in the link-layer approach $([6,7])$, which resorts to proper management of radio resources (e.g. power allocation) and error recovery to hide propagation impairments as much as possible to upper layers. This approach allows to avoid any modification at the transport layer, but at the expense of increased efforts on the lower layers of the wireless interface.

Since final performance (e.g. achievable throughput at the transport level) depends on the combined behaviour of the above mentioned components, developing consistent models that capture interactions and their complexity is of crucial relevance. Along this line, this paper moves from recent papers (e.g. ([6,7]) and proposes significant extensions in order to jointly consider the following compo-

\author{
K. H. Johansson, N. Möller \\ Royal Institute of Technology, \\ Department of Signal, Sensors and Systems, \\ SE-100 44 Stockholm, \\ Sweden \\ \{kallej, niels\}@s3.kth.se
}

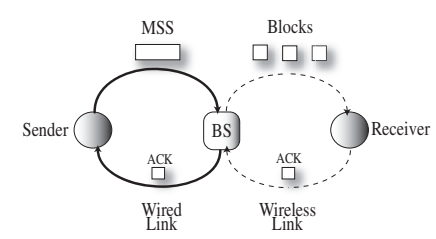

Figure 1: A model for a hybrid wired/wireless connection

nents in the optimization task: power settings (allocation), modulation format (from MSK to 8-PSK within the EDGE frame), and error recovery strategy (FEC, ARQ and Hybrid ARQ of Type I). Moreover, we account for typical error floor induced by Rayleigh fading statistics.

The outline of this paper is as follows. In section II the system model is presented, in section III the analysis of the objective function is described, while numerical results are exposed in section IV; finally in section V conclusions are drawn.

\section{SYSTEM MODEL}

Moving from [6], the transmission scheme for TCP extended over a wireless link is shown in Fig. 1. We consider only one sender, one receiver and one base station for acknowledgements (ACKs) crossing. While we do not deal specifically with fragmentation, we assume that a TCP packet of size $M S S$ (expressed in bits) is split into $J$ (radio) blocks, with $J=\frac{M S S}{K}$, where $K$ denotes the block length. Each block is then encoded and transmitted, as shown in Fig.1. Furthermore, it has been assumed that the base station has a large enough buffer.

\section{A. $T C P / I P$}

For our computations, we use the Reno version of TCP since it is the most popular implementation in the Internet today, $[8,9]$. For this reason, we consider not only the behaviour of TCP fast retransmit mechanism, but also the effects of TCP timeout mechanism, as expected from Reno congestion control algorithm, [10,11]. This latter aspect is of fundamental importance, since it has been demonstrated that in a wireless context the number of timeout events is usually larger then the number of fast retransmit events, $[12,13,14]$.

We retain in this context the following approximate expression for TCP Reno's [15]: 


$$
f\left(P_{S}\right) \approx \min \left(\frac{W_{\max }}{R T T}, \frac{1}{R T T \sqrt{\frac{2 P_{S}}{3}}+T_{0} \min \left(1,3 \sqrt{\frac{3 P_{S}}{8}}\right) P_{S}\left(1+32 P_{S}^{2}\right)}\right)
$$

where $W_{\max }$ is the maximum allowed congestion window size, $P_{S}$ is the probability of a TCP segment loss, $T_{0}$ is the Time Out period and RTT is the round trip time, whose expression will be computed in the following.

The probability that a TCP segment is discarded because of link errors in the wireless channel can be evaluated as: $P_{S}=1-\left(1-P_{\text {block }}\right)^{J}$.

\section{B. Link layer error control: Hybrid ARQ of Type I}

We assume a Hybrid ARQ (Automatic Repeat Request) of Type I [16] for error recovery at the link layer. This scheme uses a code, i.e. an $(n, k)$ linear code, which is designed for simultaneous error detection and error correction (FEC, Forward Error Correction). When the received block is detected with errors, the receiver first attempts to locate and correct the errors. If the presence of an error pattern is detected that exceeds correction capabilities, the receiver rejects the received block and requests a retransmission. For our computations we adopted a Reed-Solomon (RS) code, [16] while a Stop-and-Wait (SW) algorithm was adopted for the ARQ component.

\section{1) Encoding and FEC component}

Each $K$ bits-block is encoded in a code word of length $N$. The redundancy ratio is defined as: $x=(N-K) / K$. We assume that the probability of undetecting a corrupted block is almost zero. We also assume that ACK are well protected and there is no probability to lose them in any link. A TCP segment is decoded properly if all blocks are received uncorrupted and decoded by the receiver. In general, for an $(N, K)$ code, the coding gain, which is the reduction in the transmission power that is achieved when using a coded signal, is given by $G_{\text {coding }}=\frac{K}{N} d_{\text {min }}$. For a RS encoding technique ([16]) it results $d_{\min }=(N-K+1)$, so that it turns out: $G_{\text {coding }}=\frac{1}{x+1}(K x+1)$.

\section{2) $A R Q$ component}

If FEC does not succeed in decoding one block, the link level error recovery mechanism turns to ARQ and tries to re-send the block (frame) for $\delta$ times where $\delta$ is the maximum number of allowable retransmissions and is called persistency. If the block (frame) cannot be delivered after $\delta$ trials, ARQ assumes that the frame cannot be locally recovered and leaves to TCP the correction of the frame on an end-to-end basis. As already evidenced, we assume a SW ARQ mechanism.

\section{3) Power control}

Power control in intended as power allocation to counteract large scale (path loss) and medium scale (shadowing) fluctuations in the channel gain. This is reasonable in those systems where fast power control is not implemented. Fast fading is however accounted for, as explained in the following section, in the expression of the average bit error rate (BER) through fast fading statistics.

\section{Model for TCP throughput}

According to [6], the expression for TCP throughput can be written as follows:

$$
\lambda(x, y, \delta)=\frac{1}{x+1} \cdot f\left(R T T, P_{S}\right)
$$

where $y$ represents the transmission power.

\section{1) RTT computation}

In order to evaluate the expression for RTT, we assume that data blocks are quickly acknowledged by ARQ-SW and sizes of acknowledgments are negligible if compared to data blocks. The RTT is obviously dependent on the number of retransmissions. Let $\delta_{i}, i=1 \ldots J$ denotes the number of times we retransmit the block $i$ of a TCP segment. $\delta_{i}$ are i.i.d. geometric random variables. Therefore, the RTT mean value can be derived as follows:

$$
\begin{aligned}
E(R T T) & =T+2 D+T_{s}+\left(2 D+T_{b}\right) \sum_{i=0}^{J} E\left(\delta_{i}\right)= \\
& =T+2 D+T_{s}+\left(2 D+T_{b}\right) J E\left(\delta_{i}\right)
\end{aligned}
$$

where $T$ is the round trip time of the wired part of the TCP connection, $D$ is the one way propagation delay, $T_{b}=N / B$ is the time needed for the transmission of one block, $T_{s}=$ $J N / B=\operatorname{MSS}(x+1) / B$ is the time needed for the transmission of a segment.

\section{2) Average number of retransmissions}

The average number of retransmissions can be computed by considering that the random variables $\delta_{i}$ assume values within the range $0, ;, \delta$. It can be shown that

$$
E\left(\delta_{i}\right)=\sum_{j=0}^{\infty} j P\left(\delta_{i}=j / \delta_{i} \leq \delta\right)
$$

Moreover, it can be shown that

$$
E\left(\delta_{i}\right)=\sum_{j=0}^{\delta} j \frac{P\left(\delta_{i}=j\right)}{P\left(\delta_{i} \leq \delta\right)}
$$

and, since Reed-Solomon codes are used, [19]:

$$
P_{\text {Block }}=\left(1-\left(1-P_{b}\right)^{K}\right)^{\delta+1}
$$

where $P_{b}$ is the probability of a bit error. By assuming symbol-to-symbol independence when dealing with error events, it turns out that:

$$
P\left(\delta_{i}=j\right)=P_{\text {Block }}^{j}\left(1-P_{\text {Block }}\right)
$$

and it is possible to demonstrate that:

$$
P\left(\delta_{i} \leq \delta\right)=1-P_{\text {Block }}^{\delta+1}
$$

The following expressions are then obtained:

$$
E(\delta)=\frac{P_{\text {Block }}\left(1-P_{\text {Block }}\right)}{1-P_{\text {Block }}^{\delta+1}} \sum_{j=0}^{\delta} j P_{\text {Block }}^{j-1}
$$


where

$$
\begin{aligned}
\sum_{j=0}^{\delta} j P_{\text {Block }}^{j-1} & =\frac{\partial}{\partial P}\left(\frac{1-P_{\text {Block }}^{\delta+1}}{1-P_{\text {Block }}}\right)= \\
& =\frac{-(\delta+1) P_{\text {Block }}^{\delta}\left(1-P_{\text {Block }}\right)+\left(1-P_{\text {Block }}^{\delta+1}\right)}{\left(1-P_{\text {Block }}\right)^{2}}
\end{aligned}
$$

and finally:

$$
E\left(\delta_{i}\right)=\frac{P_{\text {Block }}}{\left(1-P_{\text {Block }}\right)}-\frac{(\delta+1) P_{\text {Block }}^{\delta+1}}{\left(1-P_{\text {Block }}^{\delta+1}\right)}
$$

\section{Definition of the objective function}

We have to consider two main aspects for the evaluation of the objective function: the throughput produced $\lambda$ and the cost to obtain it $c$. According to [6], the following expression is adopted:

$$
\gamma(x, \delta, y)=\frac{\lambda(x, \delta, y)}{c(x, \delta, y)}
$$

\section{1) Cost evaluation}

Two cost components are considered, namely [6]: a term which takes energy consumption into account and a term which considers the amount of wireless resources employed. If $x$ is the redundancy ratio introduced by FEC and if the aim is to transfer $S$ segments of $M S S$ bits size, each segment would be transmitted in a total of $J$ codewords of length $K(1+x)$ over the wireless link, where each codeword could be retransmitted $0 \leq \delta_{i} \leq \delta$ times. It follows that $S \cdot \operatorname{MSS} \cdot(1+x) \cdot\left(1+\delta_{i}\right)$ bits must be transmitted. Accordingly, the cost of the resources required to complete the transfer is given by:

$$
c_{r e s}=K_{r e s} \cdot S \cdot \operatorname{MSS} \cdot(1+x) \cdot\left(1+E\left(\delta_{i}\right)\right)
$$

where $K_{\text {res }}$ (expressed in $\mathrm{bit}^{-1}$ ) is a constant which represents the cost of the bandwidth resources required to transfer a bit. For what concerns the energy, let $K_{e n}$, expressed in Joule ${ }^{-1}$, denote the cost of a unit of energy. Considering that $y / B$ is the energy transmitted per bit, the total energy cost can be expressed as:

$$
c_{e n}=K_{e n} \cdot S \cdot \operatorname{MSS} \cdot(1+x) \cdot\left(1+E\left(\delta_{i}\right)\right) \cdot y / B
$$

It follows that the cost of the transfer of $S$ TCP segments can be evaluated as:

$$
\begin{aligned}
c(x, y) & =c_{\text {en }}+c_{\text {res }}= \\
& =S \cdot M S S \cdot(1+x) \cdot\left(K_{\text {en }} \cdot y / B+K_{\text {res }}\right) \\
& =S \cdot(x+1) \cdot\left(K_{1} y+K_{2}\right)
\end{aligned}
$$

\section{ANALYSIS OF THE OBJECTIVE FUNCTION}

In order to analyze the behaviour of the objective function we consider two modulation formats, namely Gaussian minimum shift keying (GMSK) and 8-phase shift keying (8PSK), that are included in the set of allowed formats within EDGE [18]. Moreover, in order to increase the complexity of our evaluation setup, we consider first an AWGN channel and then a Rayleigh flat fading channel.
A. Case of additive white Gaussian noise (AWGN) channel

The probability of a symbol error for the 8PSK over a link affected only by AWGN can be expressed as follows, [19]:

$$
P_{E}=\operatorname{erfc}\left(\sqrt{\frac{E}{N_{0}}} \sin \left(\frac{\pi}{8}\right)\right)=2 Q\left(\sqrt{\frac{2 E}{N_{0}}} \sin \left(\frac{\pi}{8}\right)\right)
$$

where $E$ is the energy per symbol, $N_{0}$ is the noise power spectral density and 8 is the size of the alphabet used for coding. By considering the following relations: $E=$ $\frac{P_{R}}{B} \log _{2}(8)$ and $P_{R}=A y G_{c}$, where $A$ is the path loss and $B$ is the bit rate, it follows that:

$$
P_{E}=\operatorname{erfc}\left(\sqrt{\frac{A y}{N_{0} B} \frac{K x+1}{1+x} \log _{2}(8)} \sin \left(\frac{\pi}{8}\right)\right)
$$

Assuming a Gray encoding yields:

$$
P_{b} \approx \frac{P_{E}}{\log _{2}(8)}
$$

For GMSK modulation, the following expression holds true [19]:

$$
P_{E}=\operatorname{erfc}\left(\sqrt{\frac{E}{2 N_{0}}}\right)
$$

where: $E=\left(2 A y G_{c}\right) / B$ and, when a Gray code is used, the bit error probability can be approximated as follows :

$$
P_{b}=\frac{1}{2} \operatorname{erfc}\left(\sqrt{\frac{A y}{N_{0} B} \frac{K x+1}{1+x}}\right)
$$

\section{B. Case of non-selective Rayleigh fading channel}

Let us assume a coherent detection. Following [19], it is possible to demonstrate that the bit error probabilities for the examined modulation formats can be expressed as follows. For the GMSK:

$$
P_{b}=\frac{1}{2}\left[1-\sqrt{\frac{\rho_{b}}{1+\rho_{b}}}\right]
$$

where

$$
\rho_{b}=\frac{A y G_{c}}{N_{0} B} 2 \sigma^{2}
$$

and $\sigma^{2}=E\left(\alpha^{2}\right) / 2=1 / 2$. For the 8PSK:

$$
P_{b}=\frac{1}{\log _{2}(8)}\left[1-\sqrt{\frac{\rho_{b}}{1+\rho_{b}}}\right]
$$

where

$$
\rho_{b}=\frac{A y G_{c}}{N_{0} B} \log _{2}(8) \sin ^{2}\left(\frac{\pi}{8}\right) 2 \sigma^{2}
$$

\section{NUMERICAL RESULTS}

For our computations we use the set of values presented in Table I. It has to be noticed that, besides the already mentioned extensions, we further specify with respect to previous works $[6,7]$ that a fixed radio-frequency bandwidth is assumed here, which obviously restricts the set of feasible combinations for joint settings of various parameters. Certainly, this assumption accounts for actual operations of radio systems like GSM and EDGE, that operate with a fixed $200 \mathrm{kHz}$ radio frequency bandwidth. 
Table 1: Numerical values assumed in computations.

\begin{tabular}{|l|l|}
\hline$f$ & $1800 \mathrm{MHz}$ \\
\hline$d$ & $150 \mathrm{~m}$ \\
\hline$N_{0}$ & $1.379 e(-15)$ (AWGN) \\
& $1.379 e(-20)$ (fading) \\
\hline$B$ & $\begin{array}{l}270 \mathrm{kbps} \text { (GMSK) } \\
810 \mathrm{kbps} \text { (8PSK) }\end{array}$ \\
\hline$M S S$ & 128 byte \\
\hline$K$ & 260 bit \\
\hline$T$ & $100 \mathrm{~ms}$ \\
\hline$D$ & $10 \mathrm{~ms}$ \\
\hline$T_{0}$ & $4 \mathrm{~s}$ \\
\hline$W_{\max }$ & 128 segments \\
\hline$K_{1} / K_{2}$ & 100 with $K_{2}=1$ \\
\hline
\end{tabular}

\section{A. Case of AWGN channel}

In Fig. 2 we observe that the objective function, defined in 11 , first increases to a maximum value and then reduces with increase in $x$ for each value of the transmission power; while concerning with this behaviour, we usually remark that the FEC has cleaned the radio link from TCP point of view, [7]. Moreover, we note an increase of the objective function with the increase of the transmission power, even if also in this case there is a limit. In fact from Fig. 2 we observe that we can increase the value of $y$ up to a certain optimum, beyond which $\gamma$ starts to decrease because the gain in the objective function is outweighted by the increased cost of utilized resources.

In Fig. 3 we plot the objective function obtained with the introduction of the hybrid ARQ. We observe that the maximum achievable value of $\gamma$ increases as $\delta$ increases and less redundancy is needed. We have also demonstrated that it not possible to have additional improvements by only increasing $\delta$ For what concerns 8PSK the reaction to the introduction of the hybrid ARQ is the same as for GMSK (see fig. 4,5), while we observe that the maximum of $\gamma$ is achievable for a smaller value of $x$ even if the performance is strongly reduced and the transmission power required is higher with respect to the GMSK case. These latter behaviour depends on the increased bit error probability of the multilevel modulation.

\section{B. Case of non-selective fading channel}

In Fig. 6 we plot the behaviour of the objective function in a non selective Rayleigh fading channel. We observe that the maximum achievable value of $\gamma$ is much smaller then in the ideal case, because the channel quality is worse. Moreover, the value of redundancy corresponding to the maximum of $\gamma$ does not change considerably with an increase in transmission power, while there are vertical translations.

Fig. 7 shows that the introduction of the hybrid ARQ brings important improvement in the objective function's performance. Moreover these improvements are larger then the corresponding ones produced in the ideal case of AWGN channel. This is due to the ability of the ARQ to mask the real channel quality, making TCP to experiment a lower error probability which is a critical parameter for FEC alone. Similar results have been obtained for 8PSK (see Fig. 8, 9).

We notice that in this latter case the differences between the results obtained for the two modulation formats for transmission through a fading channel are smaller then the ones previously obtained for the AWGN channel. This is due to the channel contribution $\sigma$ to the error probability which is independent on the modulation used.

\section{CONCLusions}

In this paper we have provided a performance analysis setup for TCP over wireless: it includes joint combination of hybrid ARQ, power management and modulation format in order to optimize a cost-benefit functional at the transport level. The analytical setup also captures some features of fading channels, while numerical results clearly evidenced that a joint careful tuning of all components is required in order to achieve maximum performance.

While in this paper we have been concerned with optimization in a static environment, current work is focused on actual introduction of the dynamic behavior in a mobile environment, where adaptivity can be truly exploited in varying the optimization setup during system operations and can be eventually formalized in cross-layered architectures. Future work also includes behaviours of UMTS WCDMA wireless interface and the consequent MAI (Multiple Access Interference) component induced by multi-users operations.

\section{REFERENCES}

[1] Jean-Paul M. G. Linmartz, "Wireless Communication, The Interactive Multimedia CD-ROM", Baltzer Science Publishers, P.O.Box 37208, 1030 AE Amsterdam, ISSN 1383 4231, Vol. 1 (1996), No.1.

[2] M Zorzi, R. R. Rao, L. B. Milstein, "Error statistics in data transmission over fading channels", IEEE transactions on communications, Vol. 46, No. 11, November 1998.

[3] I. Cabrera Molero, N. Möller, J. Petersson, R. Skog, A. Arvidsson, O. Flärdh, K. E. Johansson, "Cross-Layer Adaptation for TCP-Based Applications in WCDMA Systems", IST Mobile and Wireless Communication Summit, 2005.

[4] O. Tickoo, V. Subramanian, S. Kalyanaraman, K. K. Ramakrishnam, "LT-TCP: End-to-end framework to improve TCP performance over networks with lossy channels", Lecture notes in computer science, publisher: Splinger-Verlag GmbH, vol. 3552/2005, chap: p.81.

[5] S. Mascolo, C. Casetti, M. Gerla, M. Y. Sanadidi, R. Wang, "TCP Westwood: Bandwidth Estimation for Enhanced Transport over Wireless Links", MOBICOM, 2001.

[6] D. Barman, I. Matta, E. Altman, R. E. Azouzi, "TCP Optimization through FEC, ARQ and Transmission Power Tradeoffs", Technical Report, December 3, 2003.

[7] L. Galluccio, G. Morabito, S. Palazzo,"An Analytical Study of a Tradeoff between Transmission Power and FEC for TCP optimization in Wireless Networks", IEEE INFOCOM, 2003.

[8] W. Stevens, "TCP/IP illustrated", vol.1 "The Protocols", Addison-Wesley, 1994.

[9] W. Stevens, "TCP Slow Start, Congestion Avoidance, Fast Retransmit, and Fast Recovery Algorithms", RFC2001, January 1997. 


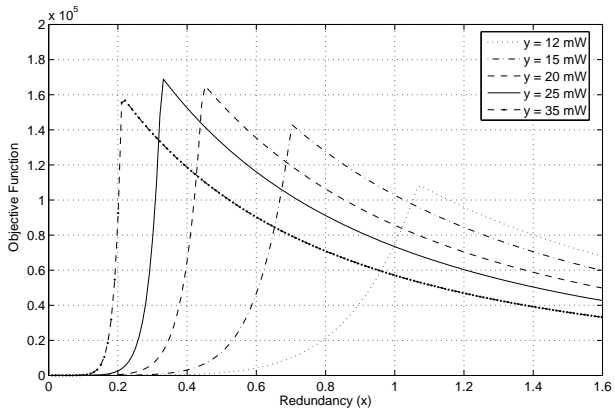

Figure 2: $\gamma$ for GMSK with FEC alone $(\delta=0)$ in a AWGN channel.

[10] Information Sciences Institute University of Southern California," RFC 792 (RFC792)-Transmission control Protocol",Internet RFC/STD/FYI/BCP Archives, September 1981.

[11] J. Postel, "RFC 792 (RFC792) - Internet Control Message Protocol", September 1981.

[12] E. Hossain, D. I. Kim, B. K. Bhargava, "Analysis of TCP Performance Under Joint Rate and Power Adaptation in Cellular WCDMA Networks", ieee transactions over wireless communications, vol. 3, no. 3, May 2004.

[13] C. Fischione, F. Graziosi, F. Santucci, N. Möller, K. H. Johansson, H. Hjalmarsson, "Analysis of TCP over WCDMA wireless systems under power control, MAI and link level error recovery", Proc. of IWCT'05, Oulu, Finland, 2005.

[14] N. Möller, K. H. Johansson, H. Hjalmarsson, "Making retransmission delays in wireless links friendlier to TCP", 43rd IEEE Conference on Decision and Control December 14-17, 2004 Atlantis, Paradise Island, Bahamas.

[15] J. Padhye, V. Firoiu, D. Towsley, J. Kurose, "Modeling TCP Throughput: A Simple Model and its Empirical Validation",in Proceeding of ACM/SIGCOMM'98, Vancouver, Canada, October 1998.

[16] S. Lin, D. J. Costello jr., "Error Control Coding: Fundamental and Applications", Prentice Hall International Editions, 1983.

[17] C. Fischione,"TCP and Radio Resource Management", Draft, July 142004.

P. Di Marco, "Studio e sviluppo di modelli per l'analisi delle prestazioni di protocolli TCP su sistemi radio", Tesi di Laurea, Università degli studi dell' Aquila, Facoltà di Ingegneria, A.A. 2004-2005.

[18] EDGE: Introduction of hi-speed data in GSM/GPRS networks White Paper Ericsson AB 2003.

[19] J. G. Proakis,"Communication Systems Engineering", Prentice Hall International Editions, 1994.

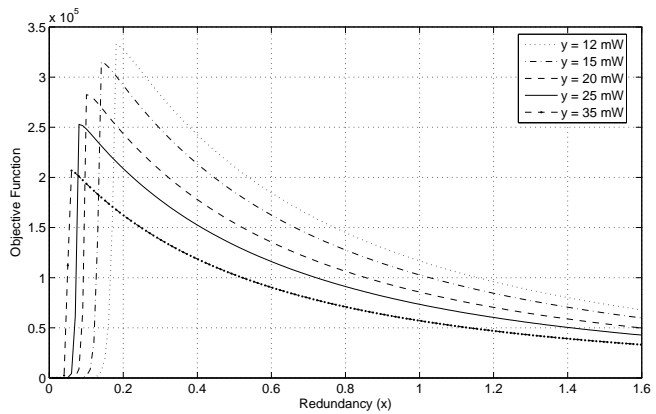

Figure 3: $\gamma$ for GMSK with hybrid ARQ $(\delta=4)$ in a AWGN channel.

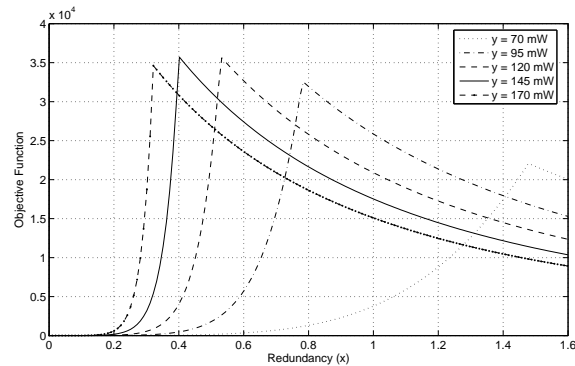

Figure 4: $\gamma$ for 8PSK with FEC $(\delta=0)$ in a AWGN channel.

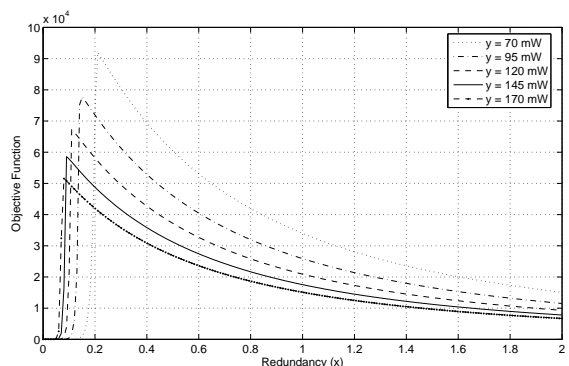

Figure 5: $\gamma$ for 8PSK with hybrid ARQ $(\delta=4)$ in a AWGN channel.

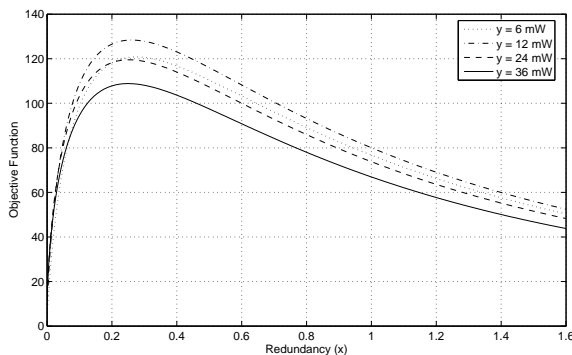

Figure 6: $\gamma$ for GMSK with FEC $(\delta=0)$ in a Rayleigh fading channel. 


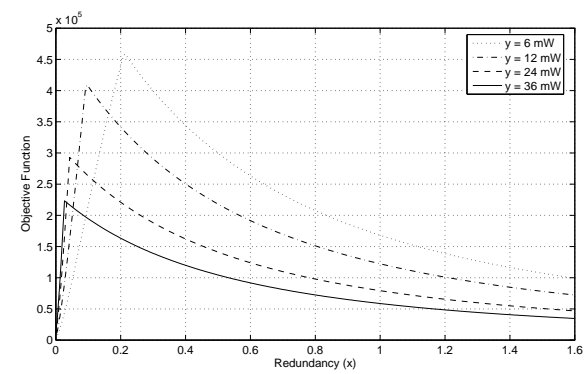

Figure 7: $\gamma$ for GMSK with hybrid ARQ $(\delta=4)$ in a Rayleigh fading channel.

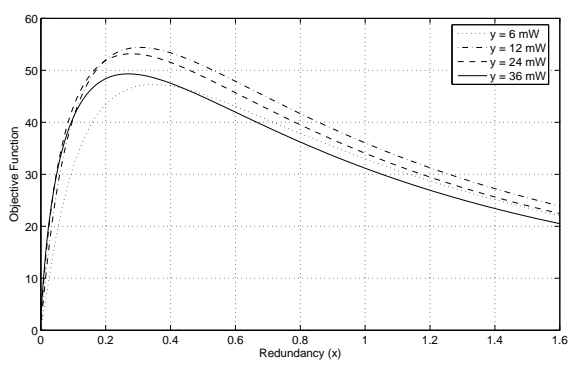

Figure 8: $\gamma$ for 8PSK with FEC alone in a Rayleigh fading channel.

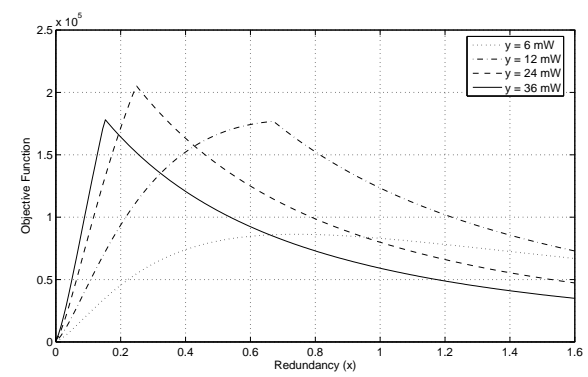

Figure 9: $\gamma$ for 8PSK with hybrid ARQ $(\delta=4)$ in a Rayleigh fading channel. 